\title{
“TO STUDY THE RELATIONSHIP OF ORAL HYGIENE AND GINGIVITIS WITH THE INFLUENCE OF TOOTH BRUSHING HABITS IN CHILDREN OF MEERUT DISTRICT"
}

Natasha Gambhir, Usha Rehani, Abhay Agarwal, Shourya Sharma

1. Senior Lecturer, Department of Pedodontics \& Preventive Dentistry, Santosh Dental College, Ghaziabad

2. Prof \& HOD, Department of Pedodontics \& Preventive Dentistry, Inderprastha Dental College, Sahibabad.

3. Prof \& HOD, Department of Pedodontics \& Preventive Dentistry, Kalka Dental College. Meerut Bypass.

4. Senior Lecturer, Department of Conservative Dentistry \& Endodontics, New Horizan Dental College, Bilaspur

\section{CORRESPONDING AUTHOR}

Dr. Natasha Gambhir,

1/6 Ram Kishor Road,

Civil lines, Delhi,

E-mail: tash_aa@yahoo.com,

Ph: 00919871966965.

ABSTRACT: Oral hygiene as a means and an end is a relatively novel concept which has only attained tactic public recognition as a desirable cosmetic endeavor during this last century. Numerous factors may affect the distribution of plaque and gingivitis in the mouth. Of considerable importance must be the oral hygiene habits of each person, which will be influenced by compliance and dexterity with tooth cleaning methods. Most people exercise some measure of oral hygiene, and tooth brushing seems to be the method of choice providing thorough and regular cleaning. AIMS \& OBJECTIVES: This present study was conducted to investigate the effect of different brushing habits on dental plaque accumulation and gingival health of children of Meerut district. RESULTS: The results of the study revealed that plaque index and modified gingival index was not affected by sex and residing status of the children. Toothbrush was observed to be the best brushing aid, followed by name datum and then finger. Further, it was found that children who used toothpaste as a dentifrice showed lesser degree of plaque and gingivitis as compared to children who used toothpowder and those who used no dentifrice. The scores were the highest in children who were using raakh as a dentifrice. Brushing once or twice daily proved to be the best frequency of brushing, followed by alternate days, occasionally and the highest for children who did not brush. It was observed that plaque was found to be directly related to gingivitis irrespective of the sex, residing status, type of brushing aid, dentifrice used and frequency of brushing. Therefore, it can be concluded that more school dental health education programs should be carried out, focusing on the need of tooth brushing using a suitable dentifrice at least once a day to maintain good oral hygiene.

KEYWORDS: Oral Hygiene, Gingivitis, Tooth brushing, Children

INTRODUCTION: 'A clean tooth never decays' was a forceful proverb in the early years of oral hygiene promotion. As we commence a new millennium the prospect of maintaining the natural dentition in relative health for a lifetime is a reasonable and achievable goal. There are several general reasons for this optimism; improved socio-economic status, better education, increased public interest in maintaining good oral health and better oral hygiene.

Gingivitis is still prevalent, but less extensive and less severe than expected. 
Early morphological and histopathological research disclosed that an intimate spatial relationship existed between dental plaque and the gingival tissues. ${ }^{1}$

Bacterial plaque, the primary etiological agent in gingivitis and supragingival plaque formation, appears to be necessary for the initial colonization of the subgingival environment. Gingivitis is inflammation of the gingiva that does not result in clinical attachment loss. ${ }^{2}$ Investigations show that marginal gingivitis starts in early childhood and its incidence and degree increase in severity in adolescence, whereas in the next decade, the incidence of gingivitis spreads insignificantly. ${ }^{3}$

The severity of gingivitis is often related to plaque accumulation which has been shown to be variable with age i.e. gingival inflammation in young children is milder than older children with similar plaque deposits.

Other factors may affect the intensity and severity of gingivitis in children, such as immunological, microbiological, and anatomic characteristics. Also the effect of demographic and socioeconomic factors on gingivitis has yet not been defined. ${ }^{3}$

Numerous factors may affect the distribution of plaque and gingivitis in the mouth. Of considerable importance must be the oral hygiene habits of each person, which will be influenced by compliance and dexterity with tooth cleaning methods. ${ }^{4}$

Gingivitis develops in individuals after $7-21$ days in absence of personal plaque removal, providing strong evidence for recommending at least daily tooth brushing. ${ }^{5}$

Oral hygiene practices in India are deeply based on tradition and culture with use of indigenous substances being widely prevalent. Most people exercise some measure of oral hygiene, and tooth brushing seems to be the method of choice providing thorough and regular cleaning. ${ }^{6}$

Nowadays, wide variations exist in type of brushing aid used, tooth brushing technique, dentifrice use, frequency and time of tooth brushing. Individual tooth brushing habits are usually consistent. Factors affecting the efficacy of tooth brushing include the technique, frequency, duration, brush type and design and the dentifrice used.7This present study was conducted to investigate the effect of different brushing habits on dental plaque accumulation and gingival health of children of Meerut district.

MATERIAL AND METHOD: The present study was conducted on a sample of four hundred and fifty nine child patients irrespective of sex and socioeconomic status between the ages of $8-14$ years studying in various schools of Meerut district and visiting the O.P.D of Department of Pedodontics and Preventive Dentistry, Subharti Dental College, Meerut.

ASSESSMENT OF VARIOUS BRUSHING HABITS: A case sheet was prepared which was made to include the vital statistics of the child patient along with his dentition All subjects were requested to answer a set of questions prepared to assess the oral health behavior of the child including the brushing activity such as frequency, brushing aid, dentifrice used, method of brushing and any other oral hygiene aid used.

EXAMINATION OF THE CHILD PATIENTS: The child patients were examined with a mirror and explorer in a sitting position. The evaluation or scoring was done on the entire dentition (whole mouth basis). The teeth were dried with an air syringe prior to the plaque index recording. 
The plaque and gingival status of each child patient was assessed using Plaque Index (Silness \& Loe, 1964)\& Modified Gingival Index ( Lobene et al., 1986)

The data was recorded according to the methodology and was put on statistical analysis to find the possible correlation amongst the different variables of study and to get a statistically significant result.

OBSERVATIONS: The children selected for this study were divided into various groups based on the sex, residing status, brushing aid, dentifrice used and frequency of brushing.

While assessing the significant difference between the sex, residing status, various types of brushing aids, dentifrice used and frequency of brushing, it was seen that plaque index and modified gingival index was not affected by sex and residing status of the children.

A significant difference was observed for various kinds of brushing aids used. Toothbrush was observed to be the best brushing aid giving relatively less scores for plaque and modified gingival indices, followed by neem datun, finger and the highest for children who did not brush.

Further, for the type of dentifrices used, it was found that children who used toothpaste as a dentifrice showed lesser degree of plaque and gingivitis as compared to children who used toothpowder and those who used no dentifrice. However the scores were the highest in children who were using raakh as a dentifrice.

Also, for frequency of brushing, statistically no significant difference was observed between those who used to brush once and twice daily. However, brushing twice daily proved to be the best frequency of brushing as it gave relatively less scores for plaque and modified gingival indices, followed by once daily, alternate days, occasionally and the highest for children who did not brush.

While finding correlation between plaque index and modified gingival index for various brushing aids, dentifrice used, frequency of brushing, sex and residing status, a positive correlation was observed between plaque indexes and modified gingival index for the sexes, residing status, various brushing aids, various dentifrices used and frequency of brushing

DISCUSSION: Oral hygiene and personal hygiene are about the cheapest form of preventive health measure. Though cheap, it is surprisingly one of the most ignored in practice especially in the under-privileged rural communities.

It has been shown that dental plaque is the predominant cause of gingivitis in man. ${ }^{1}$ The introduction of preventive dental care, including plaque removal, has been shown to decrease the level of gingivitis. ${ }^{7}$ Taani DS, Al-Wahadni AM, Al Omari M. ${ }^{8}$ in their study to evaluate the impact of toothbrushing on dental plaque concluded that more emphasis should be placed on proper oral hygiene. Wunderlich RC, Caffesse RG, Morrison EC, Temple HJ, Kerschbaum W9 concluded that tooth brushing has a therapeutic effect on naturally occurring gingivitis, enhanced by oral hygiene instructions.

The present study was conducted to investigate the effect of different tooth brushing habits on dental plaque accumulation and gingival health of children of Meerut district. It was seen that plaque index and modified gingival index was not affected by sex of the children. Okolo et al, ${ }^{10}$ reported that there was no significant difference between the plaque indices of males and females. On the contrary, Barnie T James, Leske Gary S, Ripa Louis $\mathrm{W}^{11}$ reported that females had a better oral hygiene as well as gingival status when compared with the males. 
While assessing the significant difference between the residing status, it was seen that plaque index and modified gingival index was not affected by residing status of the children. There can be several general reasons for this; improved socio-economic status, better education, increased public interest in maintaining good oral health, increased capabilities on the part of the dental professionals in delivering state of the art preventive and therapeutic services and a strong and dynamic research enterprise able to convey new concepts and approaches to prevention and cure of disease.

Mechanical plaque control is the most effective method of controlling plaque and gingivitis. ${ }^{12}$ Tooth brushing plays a pivotal role in the defense against plaque and gingivitis. ${ }^{13}$ Fernández L.B.F, Novaes B., Feitosa A.C.R, Novaes B. ${ }^{14}$ said that tooth brushing is still the most important factor in oral hygiene and maintaining gingival health. Toothbrush was observed to be the best brushing aid giving relatively less scores for plaque and modified gingival indices, followed by neem datum, finger and the highest for children who did not brush.

The use of natural products in the prevention and treatment of oral conditions has increased recently and could be of benefit to low socioeconomic level urban and rural communities. ${ }^{14}$ Chewing sticks ( Neem Datun) may have a role to play in the promotion of oral hygiene. ${ }^{15}$ Selected clinical studies have shown that chewing sticks, when properly used, can be as efficient as toothbrushes in removing dental plaque due to the combined effect of mechanical cleaning and enhanced salivation. ${ }^{7}$ Evaluation of their effectiveness requires further research.

Tooth brushing with a dentifrice is the most widely practiced form of oral hygiene in most countries. In our case toothpaste proved to be a better material for reducing plaque and gingivitis than the toothpowder. Raakh when used as a dentifrice gave the highest scores for plaque and gingival indices, even higher when compared to children using no dentifrice, which may be attributed to the presence of harmful micro organisms in raakh.

Early reports on the relationship between frequency of the toothbrushing and the state of oral hygiene were conflicting. It is now recognized that frequency of brushing is an important influence oral hygiene. ${ }^{16}$ In our study brushing once or twice daily proved to be the best frequency of brushing as it gave relatively less scores for plaque and modified gingival indices, followed by alternate days, occasionally and the highest for children who did not brush.

The role of plaque in the etiology of gingivitis and periodontitis is well established. It was observed that plaque was found to be directly related to gingivitis irrespective of the sex, residing status, type of brushing aid, dentifrice used and frequency of brushing.

CONCLUSION: Based on the results of this study it was concluded that plaque and gingival health was not affected by sex of the children and residing status. Toothbrush was observed to be the best brushing aid, followed by neem datun and lastly finger. Toothpaste was observed to be the best dentifrice followed by toothpowder, while raakh was considered to be the least suitable dentifrice even worse than using no dentifrice. Brushing once or twice daily proved to be the best frequency of brushing as it gave relatively less scores for plaque and modified gingival indices, followed by alternate days and occasionally. It was observed that plaque was found to be directly related to gingivitis irrespective of the sex, residing status, type of brushing aid, dentifrice used and frequency of brushing. Therefore more school dental health education programs should be carried out, focusing on the need of tooth brushing using a suitable dentifrice atleast once a day to maintain good oral hygiene. 
Table 1: Distribution of children according to Gender and Residing Status

\begin{tabular}{|l|l|l|l|l|l|l|l|}
\hline \multicolumn{9}{|l|}{ GENDER } & \multicolumn{3}{l|}{ RESIDING STATUS } \\
\hline \multicolumn{2}{|l|}{ MALES } & \multicolumn{2}{|l|}{ FEMALES } & \multicolumn{3}{l|}{ RURAL } & \multicolumn{2}{l|}{ URBAN } \\
\hline 226 & $49 \%$ & 233 & $51 \%$ & 306 & $67 \%$ & 153 & $33 \%$ \\
& & & & & & & \\
\hline
\end{tabular}

Table 2: Distribution of children according to Brushing aid used

\begin{tabular}{|l|l|l|}
\hline TOOTH BRUSH & 373 & $81 \%$ \\
\hline FINGER & 53 & $11 \%$ \\
\hline NEEMDATUN & 12 & $3 \%$ \\
\hline DO NOT BRUSH & 21 & $5 \%$ \\
\hline
\end{tabular}

Table 3: Distribution of children according to Dentifrice used

\begin{tabular}{|l|l|l|}
\hline TOOTH PASTE & 329 & $72 \%$ \\
\hline TOOTH POWDER & 92 & $20 \%$ \\
\hline RAAKH & 5 & $1 \%$ \\
\hline NO DENTRIFICE & 33 & $7 \%$ \\
\hline
\end{tabular}

Table 4: Distribution of children according to Frequency of brushing

\begin{tabular}{|l|l|l|}
\hline ONCE DAILY & 357 & $78 \%$ \\
\hline TWICE DAILY & 53 & $11 \%$ \\
\hline ALT DAY & 16 & $3 \%$ \\
\hline OCCAISIONALLY & 12 & $3 \%$ \\
\hline DO NOT BRUSH & 21 & $5 \%$ \\
\hline
\end{tabular}

